\title{
PROSPECÇÃO TECNOLÓGICA DA UTILIZAÇÃO DA Spirulina platensis
}

\author{
Tácila Alcântara Mendonça*ㄹ ${ }^{1}$ Janice Izabel Druzian²; Itaciara Larroza Nunes ${ }^{3}$ \\ ${ }^{1}$ Faculdade de Farmácia, Programa de Pós-graduação em Ciência de Alimentos, Universidade \\ Federal da Bahia - UFBA - Salvador/BA - Brasil (tacila_mendonca@yahoo.com.br). \\ ${ }^{2}$ Faculdade de Farmácia, Departamento de Análises Bromatológicas, Universidade Federal da \\ Bahia - UFBA - Salvador/BA - Brasil. \\ ${ }^{3}$ Escola de Nutrição, Departamento de Ciência dos Alimentos, Universidade Federal da Bahia - \\ UFBA, Salvador/BA - Brasil.
}

\begin{abstract}
RESUMO
A Spirulina platensis é uma cianobactéria de cor verde-azulada, fonte de nutrientes com aplicabilidade ecológica, econômica e nutricional. O objetivo deste trabalho foi analisar as potencialidades e evolução tecnológica, através dos depósitos de patentes em relação à utilização da microalga Spirulina platensis, nos setores industriais. A pesquisa foi realizada a partir de palavras chaves sobre o tema, nos bancos de dados de patentes do INPI, Espacent e WIPO. Verificou-se 207 patentes a respeito desta tecnologia, sendo que o cultivo da microalga (29,5\%), indústria farmacêutica (18,9\%), indústria de alimentos (15,5\%) e área médica (14,0\%) são os principais setores de sua utilização. Os principais países detentores de pedidos de patentes são República de Móldova (74), Rússia (30), Japão (23) e Coréia (21). O Brasil possui somente 4 depósitos de patentes e 1 concedida, sendo necessários mais incentivos para aumentar o seu cenário inovativo.
\end{abstract}

Palavras Chave: Spirulina platensis, tendências tecnológicas, prospecção, patentes.

\begin{abstract}
Spirulina platensis is a blue-green cyanobacterium, nutrient source with applicability ecological, economic, and nutritional. The aim of this study was to analyze the potential and technological evolution, through patent applications on the use of Spirulina platensis, in the industrial sectors. The survey was conducted from key words on the subject in the database of the INPI, European Office, the Spacenet and WIPO. There are 207 patents regarding this technology, and the cultivation of microalgae (29.5\%), pharmaceutical industry $(18.9 \%)$, food industry $(15.5 \%)$ and medical (14.0\%) are the main sectors of use. The main countries of patent holders are the Republic of Moldova (74), Russia (30), Japan (23) and Korea (21). Brazil has only 4 patent applications and 1 granted, requiring greater incentives aimed at increasing the innovative scenario of the country.
\end{abstract}

Key words: Spirulina platensis, technological trends, prospecting, patents.

Cadernos de Prospecção - ISSN 1983-1358 (print) 2317-0026 (online), 2012. vol.5, n.1, p.44-52 
Área tecnológica: Tecnologia de alimentos, produtos naturais.

\section{INTRODUÇÃO}

Microalgas são microrganismos que crescem geralmente em meio líquido, se multiplicam rapidamente e são capazes de realizar fotossíntese oxigênica, produzindo biomassa rica em compostos biologicamente ativos. Algumas espécies de microalgas são cultivadas comercialmente como as espécies dos gêneros Chlorella, Dunaliella salina, Haematococcus pluvialis (Chlorophyceae) e Arthrospira (Spirulina) (Cyanophyceae) (DERNER et al., 2006), devido à aplicabilidade na indústria de alimentos (COLLA et al., 2004; AMBROSI et al., 2008; SILVA, 2008; CHU et al., 2010), e para a produção de aditivos naturais, tais como $\beta$-caroteno e astaxantina (DERNER et al., 2006; GUEDES et al., 2011).

A Spirulina platensis é uma cianobactéria filamentosa de cor verde-azulada, encontrada em locais como solos, pântanos, lagos alcalinos e águas salobras, marinhas e doces. Através da fotossíntese converte os nutrientes em matéria celular e libera oxigênio (COSTA et al., 2003; BERTOLIN et al., 2005; DERNER et al., 2006; AMBROSI et al., 2008). Esta microalga é composta por cerca de 6070\% de proteína, e aminoácidos específicos (ESTRADA et al., 2001; COLLA et al., 2004; AMBROSI et al., 2008) (os não essenciais - alanina, arginina, ácido aspártico, cistina, ácido glutâmico, glicina, histidina, prolina, serina e tirosina e os essenciais - isoleucina, leucina, lisina, metionina, fenilalanina, treonina e valina) (AMBROSI et al., 2008).

É constituída também por carboibratos, minerais (ferro, cálcio, fósforo, magnésio, zinco, cobre, cromo, manganês, o sódio e o potássio), vitaminas (biotina, ácido fólico, inositol, vitamina E, B12 (ESTRADA et al., 2001), B6, B3, B2, B1 e ácido pantotênico) (WANG et al., 2007; AMBROSI et al., 2008), compostos fenólicos (ácido caféico, clorogênico, salicílico, sináptico e trans-cinâmico) (ESTRADA et al., 2001; COLLA et al., 2007;), pigmentos fotossintéticos como clorofila a, luteína, $\beta$-caroteno, ficocianina, aloficocianina, dentre outros (ESTRADA et al., 2001; WANG et al., 2007; SILVA, 2008; MALA et al., 2010; MOHAMMED \& MOHD, 2011), e ácidos graxos como ácido gama-linolênico, $\alpha$-linolênico, e o araquidônico (COSTA et al. 2003; COLLA et al., 2004; COLLA et al., 2007; WANG et al., 2007; SILVA, 2008; CHU et al., 2010).

Estudos 'in vitro' e 'in vivo' mostram que as propriedades nutricionais da microalga Spirulina platensis têm sido relacionadas com possíveis propriedades terapêuticas que podem auxiliar no tratamento de problemas de saúde como diabetes, artrite, anemia, desnutrição, obesidade, tensão pré-menstrual, doenças cardiovasculares, câncer, entre outros. Por esta razão tem sido adicionada em produtos farmacêuticos, alimentares e comercializada principalmete como alimento funcional, nutracêutico e/ou suplemento alimentar, devido ao seu elevado conteúdo de proteínas, ácido gamalinolênico, vitaminas e minerais que podem melhorar a qualidade nutricional das preparações (ESTRADA et al., 2001; COLLA et al., 2004; HERRERO et al., 2005; COLLA et al., 2007;ABD EL-BAKY et al., 2007; WANG et al., 2007; AMBROSI et al., 2008; CHU et al., 2010).

Nos últimos anos verifica-se o crescente interesse pela utilização de microalgas em estudos e processos biotecnológicos, devido à aplicabilidade econômica, ecológica e nutricional, principalmente pela identificação de substâncias sintetizadas por estes organismos, que podem ter aplicabilidade comercial na nutrição, saúde humana e animal, como também no tratamento de águas residuais, produção de energia e obtenção de compostos de interesse para as indústrias alimentares, química, cosmética e farmacêutica, entre outras (COSTA et al., 2003; DERNER et al., 2006; DAO-

Cadernos de Prospecção - ISSN 1983-1358 (print) 2317-0026 (online), 2012. vol.5, n.1, p.44-52 
LUN \& ZU-CHENG, 2006; WANG et al., 2007; SILVA, 2008; AMBROSI et al., 2008; CHU et al., 2010).

Diante desse cenário, o objetivo deste trabalho foi realizar um estudo de prospecção tecnológica para avaliar o panorama mundial da utilização da Spirulina platensis, relacionando os documentos de patentes depositados sobre a utilização e aplicação da microalga, bem como estabelecer quais países são os principais detentores desta tecnologia.

\section{METODOLOGIA OU ESCOPO}

Essa prospecção tecnológica foi realizada entre os meses de agosto e setembro de 2012, tendo como base os pedidos de patentes depositados no European Patent Office (Espacenet), na World Intellectual Property Organization (WIPO) e no Banco de dados do Instituto Nacional de Propriedade Industrial (INPI) do Brasil. O foco da pesquisa foi a microalga Spirulina platensis e sua utilização e aplicação nos diversos setores industriais.

Para a obtenção dos dados foi elaborada uma estratégia de busca levando em consideração palavras-chave como o nome científico da migroalga (Spirulina platensis, Arthrospira platensis e Spirulina spp,) acrescida de alguns substantivos de interesse: alimento (food), suplemento (supplement), cultivo (cultivation), biomassa (biomass), obtenção (obtaining), composição (composition), cosmético (cosmetic), partículas (particles) e cápsulas (capsule), termos estes que poderiam representar a forma como esta microalga poderia ser encontrada nos documentos de patentes.

Foi utilizada a pesquisa avançada (Advanced Search) e os campos de pesquisa “título” e "resumo” nos bancos de patentes durante o levantamento de dados. Foram excluídos documentos pertencentes à mesma família de patentes. A prospecção tecnológica foi realizada por meio de coleta, tratamento e análise das informações extraídas dos documentos de patentes encontrados.

\section{RESULTADOS E DISCUSSÃO}

O resultado deste estudo revelou um universo total de 313 documentos de patentes referente à tecnologia de interesse, entretanto isso não representa o valor total de invenções protegidas, visto que uma patente pode ser depositada mais de uma vez em diferentes países devido ao direito da territorialidade.

Após a análise das informações extraídas dos documentos da tecnologia de interesse, 206 patentes foram incluídas no universo de dados dessa pesquisa. Foram encontradas 188 patentes na base européia - Espacenet (no campo título e resumo), 13 na WIPO (no campo folha de rosto) e 05 na base do INPI (no campo resumo, com palavras-chave em inglês).

Alguns relatos informam que o uso da Spirulina platensis na dieta humana está presente desde a pré-história, sendo consumida, por exemplo, entre os povos antigos do Lago Texcoco (México) e nativos do Lago Chade (África) (COSTA et al., 2007; COSTA \& MORAIS, 2011). Entre os anos de 1964 e 1965 pesquisadores franceses iniciaram os primeiros estudos a respeito da microalga, devido às atividades de campo realizadas no Lago Texcoco no México (LEONARD, 1966). Todavia, somente a partir da década de 1970 verificou-se um maior interesse a respeito desta

Cadernos de Prospecção - ISSN 1983-1358 (print) 2317-0026 (online), 2012. vol.5, n.1, p.44-52 
matéria-prima, devido à publicação de estudos revelando as suas propriedades nutricionais, medicinais e terapêuticas (CHAMORRO et al., 1996; AMBROSI et al., 2008).

Na Figura 1 é demonstrada a evolução anual de depósitos de patentes relacionados à utilização da Spirulina platensis, nos diversos setores industriais (cultivo, medicina, farmacêuticas, alimentos, dentre outros) entre 1971 a 2011. Entre os anos de 1975 a 1986 têm-se depósitos de pedidos voltados à tecnologia básica de obtenção da microalga, refletindo possivelmente os resultados dos estudos que associam a mesma a diversos fins comerciais, na alimentação humana e animal, no tratamento de águas residuais, produção de energia e, principalmente, na produção de compostos biologicamente ativos de interesse para as indústrias alimentares, química, cosmética e farmacêutica, dentre outras (COSTA et al., 2003; KEPEKÇI \& SAYGIDEGER, 2012).

Todavia, entre os anos de 2005 e 2007 há maior presença de pedidos de patentes (22), este resultado possivelmente pode estar associado a um maior investimento financeiro em Ciência e Tecnologia (C\&T) por parte dos países investidores, na intenção de proporcionar maiores avanços na área de pesquisa e de desenvolvimento da tecnologia de interesse. Vale ressaltar que essa ação é positiva, pois permitirá o desenvolvimento de novos processos tecnológicos, como impulsionará a pesquisa e o desenvolvimento científico a respeito da utilização da microalga em diferentes seguimentos industriais.

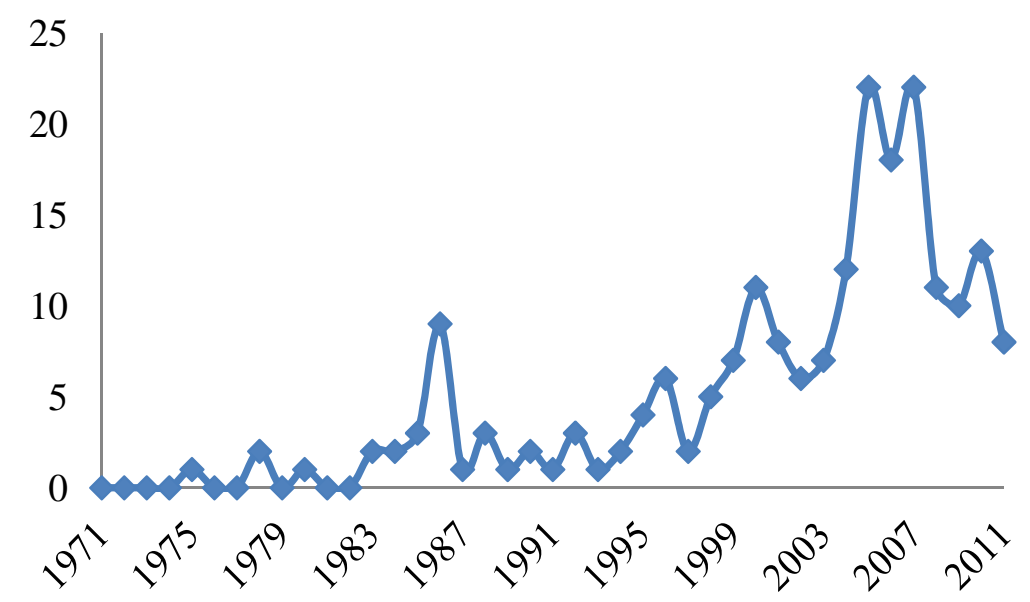

Figura 1: Evolução anual de depósitos de patentes da microalga Spirulina platensis entre 1971 e 2011. Fonte: Autoria própria, 2012.

Na Figura 2 verifica-se a distribuição de patentes de acordo a área de aplicação no setor industrial. Nota-se que as formas de cultivo da microalga (29,5\%), indústria farmacêutica (18,9\%), indústria de alimentos (15,5\%) e área médica $(14,0 \%)$ são os principais setores de utilização da microalga. Verifica-se que apenas 4,8\% destinam-se às atividades voltadas para a biotecnologia. 
O grande interesse pela forma de cultivar a microalga deve-se à possibilidade de modificar e criar meios de cultivo com diferentes substratos, a fim de proporcionar a produção de uma biomassa rica em determinados nutrientes como, por exemplo, proteínas, ácidos graxos polinsaturados, vitaminas, dentre outros, matéria-prima que poderá ter aplicabilidade nos diversos setores industriais (LÉON, 2010; BERTOLIN et al., 2005).

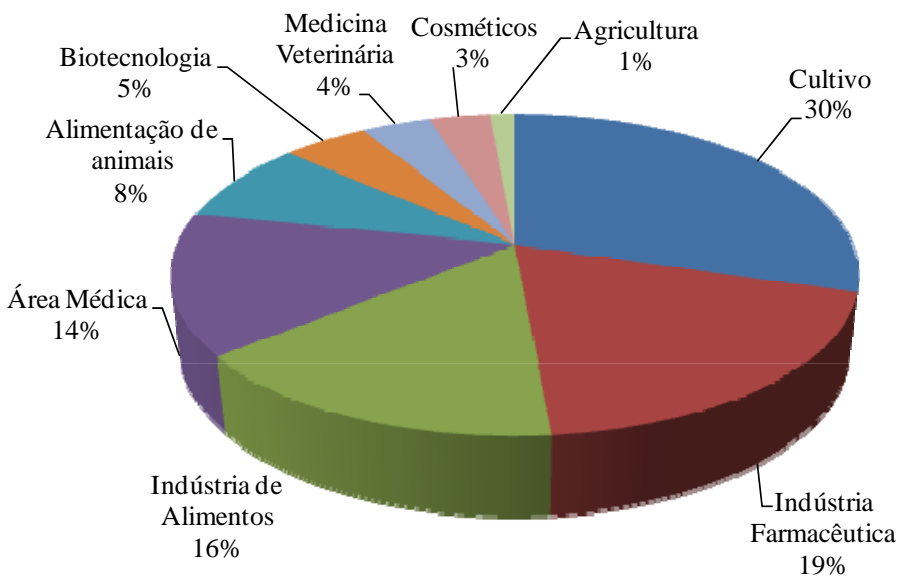

Figura 2: Distribuição de patentes de acordo área de aplicação.

Fonte: Autoria própria, 2012.

O interesse das indústrias farmacêuticas, de alimentos e área médica deve-se aos estudos 'in vitro' e 'in vivo' que revelam que a microalga Spirulina platensis apresenta propriedades nutricionais que estão relacionadas com possíveis ações terapêuticas que podem auxiliar no tratamento de problemas de saúde (ESTRADA et al., 2001; COLLA et al., 2004; HERRERO et al., 2005; COLLA et al., 2007; ABD EL-BAKY et al., 2007; WANG et al., 2007; AMBROSI et al., 2008; CHU et al., 2010).

Além disso, estes setores industriais são os principais responsáveis pelo investimento financeiro em pesquisas voltadas para ciência e tecnologia de novos produtos, uma vez que isso pode se traduzir em fins comerciais e lucrativos para o país financiador, já que uma patente pode ser depositada em diferentes países, com o objetivo de garantir o direito de exclusividade aos depositantes nos mercados considerados mais relevantes, garantindo assim o direito territorial da patente.

Os principais países detentores de pedidos de patentes utilizando a Spirulina platensis (Figura 3) são República de Moldova (74), Rússia (30), Japão (23) e Coréia (21). O Brasil apresenta apenas 4 depósitos de pedidos de patentes e 1 uma patente concedida a respeito desta tecnologia, sendo 4 desses depósitos realizados por universidades (Universidade de São Paulo, Universidade Federal do Rio Grande e Universidade Federal do Paraná) e 1 por empresa privada nacional.

As patentes brasileiras (Tabela 1) depositadas no INPI tiveram o estado de São Paulo (3) como o maior detentor das titularidades, e os pedidos de patentes estão voltados principalmente para atividades de cultivo, sobretudo para a produção de compostos específicos (como ácidos graxos, biopolímeros, proteínas, dentre outros). Cabe ressaltar que os principais centros de estudos e as

Cadernos de Prospecção - ISSN 1983-1358 (print) 2317-0026 (online), 2012. vol.5, n.1, p.44-52 
melhores condições de cultivo em nível experimental da Spirulina platensis encontram-se na região Sudeste e Sul do país, devido principalmente à infra-estrutura montada e dedicada exclusivamente para a realização de pesquisas, aperfeiçoamento e desenvolvimento de técnicas acerca do cultivo, da produção e utilização da biomassa microalgal.

Nota-se que no Brasil ainda existem poucos pedidos de patentes referentes a esta tecnologia e este resultado pode estar associado à falta de uma tradição local sobre o assunto, imaturidade do sistema de inovação, assim como a poucos incentivos do mercado brasileiro, universidades e políticas governamentais mais elaboradas capazes de promover e permitir o avanço e desenvolvimento de novas tecnologias.

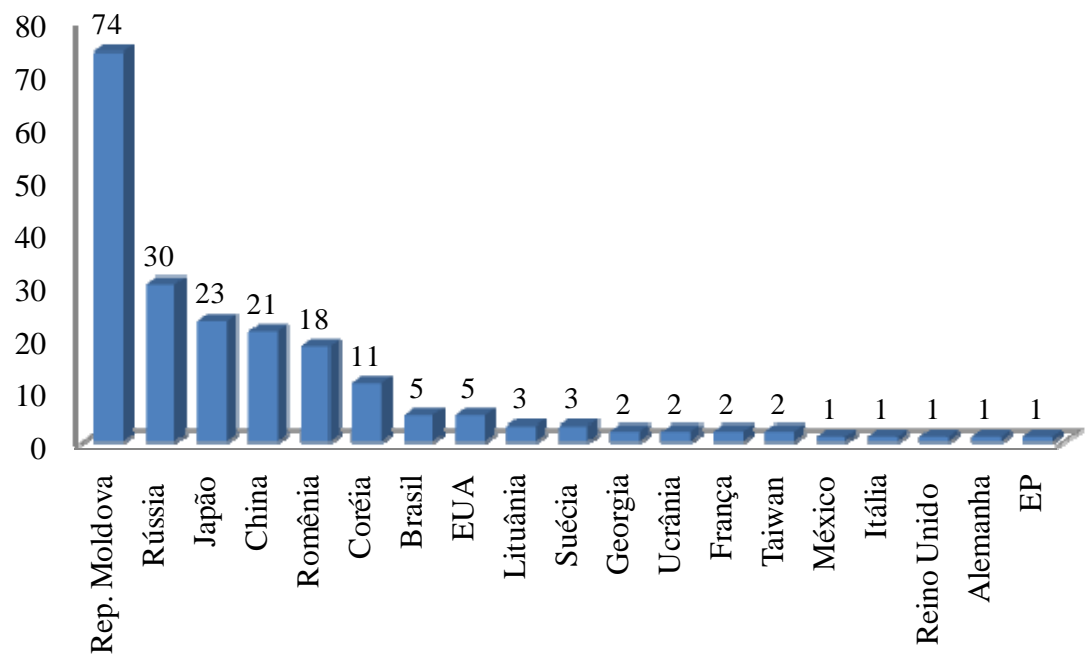

Figura 3: Patentes depositadas por país no INPI, Espacenet e WIPO. Sendo MD (República de Móldova), JP (Japão), RU (Rússia), RO (Romênia),CN (China), KR (Coréia), BR (Brasil), US (Estados Unidos), LT (Lituânia), SE (Suécia),GE (Georgia), UA (Ucrânia), FR (França), TW (Taiwan), MX (México), IT (Itália), GB (Reino Unido), DE (Alemanha), ES (Espanha). Fonte: Autoria própria, 2012

Tabela 1: Documentos de patentes no Brasil até setembro de 2012, sobre a microalga Spirulina platensis.

\begin{tabular}{lllll}
\hline Item & Número & Título da Patente & $\begin{array}{l}\text { Estado } \\
\text { Depósito/ } \\
\text { Concessão }\end{array}$ \\
\hline \multirow{1}{1}{} & $\begin{array}{l}\text { PI9003291- } \\
8\end{array}$ & $\begin{array}{l}\text { Processo de obtenção de um meio de cultura para a } \\
\text { produção de Spirulina spp }\end{array}$ & SP & $04 / 07 / 1990$ \\
2 & PI0801270- & $\begin{array}{l}\text { Processo para produção de biomassa e proteínas por } \\
\text { microalgas }\end{array}$ & SP & 08/01/2008 \\
\hline
\end{tabular}




\begin{tabular}{lllll}
\hline 3 & $\begin{array}{l}\text { PI0801702- } \\
6\end{array}$ & $\begin{array}{l}\text { Obtenção do extrato de ficocianina a partir de } \\
\text { Spirulina sp. }\end{array}$ & RS & 12/02/2008 \\
4 & $\begin{array}{l}\text { PI0805123- } \\
2\end{array}$ & $\begin{array}{l}\text { Método de aproveitamento de dióxido de carbono e seu } \\
\text { uso no cultivo de microorganismos fotossintetizantes }\end{array}$ & SP & $19 / 11 / 2008$ \\
5 & PI004637-2 & $\begin{array}{l}\text { Produtos cosméticos contendo extratos e componente } \\
\text { microalgais e proceso para a produção dos mesmos }\end{array}$ & PR & $12 / 11 / 2010$ \\
\hline
\end{tabular}

Fonte: Autoria própria, 2012.

Numa tentativa de modificar este cenário, existem grupos científicos trabalhando com a Spirulina platensis, a exemplo, temos a Rede de pesquisa da Universidade Federal do Rio Grande/RS (FURG) - REDE NANOBIOTEC (que reúne parcerias com universidades nacionais, internacionais, empresas nacionais, governamentais e não-governamentais), cujo objetivo é proporcionar a formação de uma massa crítica de recursos humanos bem como estimular o desenvolvimento de produtos de alta tecnologia a fim de difundir o tema nanofotobiotecnologia no país. Com esse estímulo e parcerias espera-se que o país desperte o interesse sobre o campo de inovações e invista financeiramente na geração de novas tecnologias.

\section{CONCLUSÃO OU COMENTÁRIOS FINAIS}

A microalga Spirulina platensis apresenta-se em potencial de crescimento para a produção de novas tecnologias, devido à aplicabilidade econômica (taxa de crescimento rápido), ecológica (cultivo simples sem degradação do meio ambiente) e nutricional (devido às substâncias que são sintetizadas pela mesma), o que representa potencial de utilização nas diversas áreas da tecnologia industrial.

As patentes depositadas nos bancos de dados estão voltadas principalmente para as áreas de cultivo, indústria farmacêutica, indústria de alimentos e médica. O Brasil apresenta poucas patentes depositadas e São Paulo é o principal estado detentor desta tecnologia. O fato de não existirem muitas patentes depositadas no Brasil pode estar relacionada à falta de cultura local a respeito do tema, bem como a falta de interesse do mercado brasileiro e incentivos que poderiam aumentar o cenário de inovações voltadas para a origem de novas tecnologias.

Para que haja o desenvolvimento de novas tecnologias no Brasil se faz necessária a difusão de informações a respeito do tema, como a combinação de políticas governamentais e estratégias empresariais mais efetivas a fim de possibilitar a criação de um ambiente propício para impulsionar a realização de atividades voltadas para a geração de inovações tecnológicas.

\section{AGRADECIMENTOS}

Os autores agradecem a CAPES pelo financiamento concedido ao projeto Rede Nanofotobiotec Rede integradora de nanotecnologia e biotecnologia microalgal para o desenvolvimento científico/tecnológico e formação de recursos humanos (AUX-PE-NANOBIOTEC 769/2009).

\section{REFERÊNCIAS}

Cadernos de Prospecção - ISSN 1983-1358 (print) 2317-0026 (online), 2012. vol.5, n.1, p.44-52 
ABD EL-BAKY, H. H.; EL BAZ, F. K.; EL-BAROTY, G. S. Enhancement of antioxidant production in Spirulina plantensis under oxidative stress. American-Eurasian Journal of Scientific Research, v. 2, n. 2, p. 170-179, 2007.

AMBROSI, M. A.; REINEHR, C. O.; BERTOLIN, T. E.; COSTA, J. A. V.; COLLA, L. M. Propriedades de saúde da microalga Spirulina. Revista de Ciências Farmacêuticas Básica e Aplicada, v. 29, n. 2, p. 115-123, 2008.

BERTOLIN, T. B. P.; COSTA, J. A. V.; BERTOLIN, T. E.; COLLA, L. M.; HEMKEMEIER, M. Cultivo da cianobactéria Spirulina platensis a partir de efluente sintético de suíno. Ciência e Agrotecnologia, Lavras,RS, v. 29, n. 1, p. 118-125, 2005.

CHAMORRO, G., SALAZAR, M., FAVILA, L., and BOURGES, H. Farmacología y toxicología del alga Spirulina. Rev Invest Clin., n. 48, p. 389-399, 1996.

CHU, W.-L.; LIM, Y.-W; RADHAKRISHNAN, A. K.; LIM, P.-E. Protective effect of aqueous extract from Spirulina platensis against cell death induced by free radicals. BCM Complementary and Alternative Medicine, v. 10, n. 53, p.1472-6882, 2010.

COLLA, L. M.; BERTOLIN, T. E.; COSTA, J. A. V. Fatty acids profile of Spirulina platensis grown under different temperatures and nitrogen concentrations. Z. Naturforsch, n. 59, 55-59, 2004.

COLLA, L. M.; REINEHR, C. O.; REICHERT, C.; COSTA, J. A. V. Production of biomass and nutraceutical compounds by Spirulina platensis under different temperature and nitrogen regimes. Bioresource Technology, n. 98, p. 1489-1493, 2007.

COSTA, J. A. V.; COLLA, L. M.; DUARTE FILHO, P. Spirulina platensis Growth in Open Raceway Ponds Using Fresh Water Supplemented with Carbon, Nitrogen and Metal Ions. Z. Naturforsch, n. 58, p. 76-80, 2003.

COSTA, J. A. V.; MORAIS, M. G. The role of biochemical engineering in the production of biofuels from microalgae. Bioresource Technology, n. 102, p. 2-9, 2011.

DAO-LUN, F.; ZU-CHENG, W. Culture of Spirulina platensis in human urine for biomass production and $\mathrm{O}_{2}$ evolution. Journal of Zhejiang University Science B, v. 7, n. 1, p. 34-37, 2006.

DERNER, R. B.; OHSE, S.; VILLELA, M.; CARVALHO, S. M. de; FETT, R. Microalgas, produtos e aplicações. Ciência Rural, Santa Maria, RS, v. 36, n. 6, p.1959-1967, 2006.

ESTRADA, J. E. P.; BESCÓS, P. B.; FRESNO, A. M. V. del. Antioxidant activity of different fractions of Spirulina platensis protean extract. Il Farmaco, n. 56, p. 497-500, 2001.

GUEDES, A. C.; AMARO, H. M.; MALCATA, F. X. Microalgae as sources of carotenoids. Marine Drugs, n. 9, p. 625-644, 2011.

HERRERO, M.; ÁlVAREZ, P. J. M.; SEÑORÁNS, F. J.; CIFUENTES, A.; IBÁÑEZ, E. Optimization of accelerated solvent extraction of antioxidants from Spirulina platensis microalga. Food Chemistry, n. 93, p. 417-423, 2005.

KEPEKÇI, R. A.; SAYGIDEGER, S. D. Enhancement of phenolic compound production in Spirulina platensis by two-step batch mode cultivation. J Appl Phycol, n. 24, p. 897-905, 2012.

Cadernos de Prospecção - ISSN 1983-1358 (print) 2317-0026 (online), 2012. vol.5, n.1, p.44-52 D.O.I.: 10.9771/S.CPROSP.2012.005.005 
LÉON, I. A. A. Estudo do cultivo de Spirulina platensis por processo contínuo com uréia como fonte de nitrogênio. 2010. 98f. Dissertação (Mestrado em Ciências Farmacêuticas) - Programa de Pós-Graduação em Tecnologia Bioquímico - Farmacêutica, Universidade de São Paulo, São Paulo, 2010.

LÉONARD, J. The 1964-65 Belgian Trans-Saharan Expedition. Nature, n. 209, p. 126-128, 1966.

MALA, R.; KARTHIK, V.; SAKTHISELVAN, S.; SARAVANABABU, S. Milking of Spirulina platensis for the production of carotenoids by aqueous two phase bioreactor.systems. Int. J. Chem. Sci., v. 8, n. 5, p. 84-91, 2010.

MOHAMMED, M. K.; MOHD, M. K. Production of carotenoids (antioxidants/ colourant) in Spirulina platensis in response to indole acetic acid (IAA). International Journal of Engineering Science and Technology, v. 3, n. 6, p. 4973-4979, 2011.

SILVA, L. A. Estudo do processo biotecnológico de produção, extração e recuperação do pigmento ficocianina da Spirulina platensis. 2008. 87f. Dissertação (Mestrado em Engenharia Química) - Programa de Pós-Graduação em Processos Biotecnológicos, Universidade Federal do Paraná, Curitiba, PR, 2008.

WANG, L.; PAN, B.; SHENG, J.; XU, J.; HU, Q. Antioxidant activity of Spirulina platensis extracts by supercritical carbon dioxide extraction. Food Chemistry, n. 105, 36-41, 2007. 